\title{
Transcriptomic and metabolomic profiling reveal the p53-dependent benzeneacetic acid attenuation of silica-induced epithelial- mesenchymal transition in human bronchial epithelial cells
}

Zhao Ju ${ }^{1}$, Jianlin Shao ${ }^{2}$, Meiling Zhou ${ }^{1}$, Jing Jin ${ }^{1}$, Huiji Pan ${ }^{1}$, Ping Ding ${ }^{1}$ and Ruixue Huang ${ }^{1 *}$

\begin{abstract}
Background: Silica exposure underlies the development of silicosis, one of the most serious occupational hazards worldwide. We aimed to explore the interaction of the silica-induced epithelial-mesenchymal transition (EMT)-related transcripts with the cellular metabolism regulated by p53.

Methods: We knocked out p53 using CRISPR/Cas9 in the human bronchial epithelial (HBE) cell line. The transcriptomic and metabolomic analyses and integrative omics were conducted using microarrays, GC-MS, and MetaboAnalyst, respectively.

Results: Fifty-two mRNAs showed significantly altered expression in the HBE p53-KO cells post-silica exposure. A total of 42 metabolites were putatively involved in p53-dependent silica-mediated HBE cell dysfunction. Through integrated data analysis, we obtained five significant p53-dependent metabolic pathways including phenylalanine, glyoxylate, dicarboxylate, and linoleic acid metabolism, and the citrate cycle. Through metabolite screening, we further identified that benzeneacetic acid, a key regulation metabolite in the phenylalanine metabolic pathway, attenuated the silica-induced EMT in HBE cells in a p53-dependent manner. Interestingly, despite the extensive p53-related published literature, the clinical translation of these studies remains unsubstantial.
\end{abstract}

Conclusions: Our study offers new insights into the molecular mechanisms by which epithelial cells respond to silica exposure and provide fresh perspective and direction for future clinical biomarker research and potential clinically sustainable and translatable role of p53.

Keywords: p53, Silica, EMT

\footnotetext{
*Correspondence: huangruixue@csu.edu.cn

${ }^{1}$ Department of Occupational and Environmental Health, Xiangya School

of Public Health, Central South University, Changsha 410078, Hunan,

China

Full list of author information is available at the end of the article
}

\begin{abstract}
Introduction
Silica, a carcinogenic substance, is most commonly associated with a variety of lung diseases including silicosis, lung cancer risk, and the development of autoimmune alteration $[1,2]$. Occupational exposure to silica in the mining industry has been observed for almost 150 years [3]. Massive inhalation of silica-based particles leads to lung fibrosis and lung dysfunction. However, at present, the rapid increase in the global population and
\end{abstract}

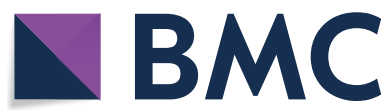

(c) The Author(s) 2021. This article is licensed under a Creative Commons Attribution 4.0 International License, which permits use, sharing, adaptation, distribution and reproduction in any medium or format, as long as you give appropriate credit to the original author(s) and the source, provide a link to the Creative Commons licence, and indicate if changes were made. The images or other third party material in this article are included in the article's Creative Commons licence, unless indicated otherwise in a credit line to the material. If material is not included in the article's Creative Commons licence and your intended use is not permitted by statutory regulation or exceeds the permitted use, you will need to obtain permission directly from the copyright holder. To view a copy of this licence, visit http://creativeco mmons.org/licenses/by/4.0/. The Creative Commons Public Domain Dedication waiver (http://creativecommons.org/publicdomain/ zero/1.0/) applies to the data made available in this article, unless otherwise stated in a credit line to the data. 
the corresponding growth in economic development have exponentially increased the number of work environments with silica exposure [4-7]. In the United States (1999-2018), the number of silicosis-related deaths decreased by $40.4 \%$ [8], whereas globally, the numbers increased by $66.0 \%$ from 36,000 to 1990 to 60,000 in 2017 [9]. China has reported the highest number $(640,000)$ of silicosis cases over the past three decades [10]. Barnes et al. noted that silica-associated silicosis is a persistent old-world occupational hazard in modern industries [11]. Although the research in the past 20 years has examined the role of silica dust in the development of silicosis, lung injury, and autoimmune diseases, our progress has been limited by the following questions: (1) How does silica exposure lead to varied prognoses and clinical characteristics?; (2) What are the potential mechanisms of lung injury caused by new sources of silica exposure?; (3) How exactly have studies tried to translate to clinic in silicainduced diseases? Our focus in this field at present is to identify evidence that was either previously ignored or missing instead of trying to approach research with mottos such as "100\% preventive" or "zero tolerance in the workplace" [12]. Consequently, a better understanding of the pathogenesis of silica-induced diseases would help discover more precise cellular targets and active signaling pathways to decipher the natural silica-associated lung diseases.

The p53 protein was discovered in 1979 [13] in normal and cancerous cells through four different groups in England, the United States and France simultaneously. Over the past 40 years, p53 has attracted considerable attention and fascination due to its fundamental and important role in gene regulation that safeguards cells against hazardous insults [14]. According to the search results in PubMed (https://pubmed.ncbi.nlm.nih.gov/), more than 100,000 hits were found for literature related to p53 (cumulative of epidemiological and molecular mechanistic research). A typical finding is that p53 function is context-dependent, dependent on the stresses, activating different signaling pathways, and cell states [15]. Indeed, more than $50 \%$ of human cancers illustrates p53 mutation, and some of mutant p53 proteins not lose activity but acquire oncogenic function [14]. p53 is also involved in silica-induced silicosis progression. Cheng et al. found that circRNA-012091 promotes lung fibroblast proliferation and migration by regulating a major member of the p53 family, PPP1R13B, in L929 cells [16]. Another study indicated that silica treatment in lung fibroblasts increased p53 expression, enhancing cell migration through activation of the p53/PUMA signaling pathway [17]. Wang et al. considered that the abnormal regulation of p53 by silica might contribute to the development of lung cancer and lung cancer [18].
Although certain studies of p53 involvement in silicainduced diseases had valuable discoveries, there remains much to learn about the roles and regulation of p53 from other new viewpoints. Recently, owing to the development of transcriptomics and metabolomics technology, an increasing number of new p53 functions have been discovered. Wang et al. indicated that a critical metabolite, 20(S)-protopanaxatriol, promoted p53 binding with DNA to form an effective anticancer network through multi-omics detection and analysis [19]. Another study also used integrative omics analysis to obtain a more in-depth understanding of p53-dependent regulation of metabolism, providing a more comprehensive view of its roles in metabolism [20]. Our previous study revealed that through integrative transcriptomics and metabolomics analysis, in response to radiation insult, p53 may dysregulate nitrogen, glutathione, and arachidonic acid metabolism, and glycolysis or gluconeogenesis [21]. This indicates the importance of p53-regulated metabolism in response to environmental toxins and their insults. Hence, we hypothesized that in the silica insulation, lung epithelial cells may be regulated through p53-dependent metabolic pathways, which may be affected by p53 and contribute to the silica-induced lung fibrosis. To verify this, the human bronchial epithelial (HBE) cell line was used to knockout p53 using CRISPR/Cas9. Integrative transcriptomic and metabolomic analyses were performed. Moreover, since p53 research is a hotspot and attracted major attention in scientific community, to further illustrate the p53 research trends globally and provide more information for translating p53 research into clinical application, we also present research and funding trends over the past 10 years.

\section{Methods and materials}

This study has three parts. The first part was the transcriptomics and metabolomics analysis, the second part was the cell mechanism experiment, and the third was the p53 research trends analysis.

\section{Cell line, construction of p53-knockout (p53-KO) cell line and multi-omics detection}

The HBE cell line was purchased from ATCC (American Type Culture Collection, USA) and stored under the conditions described in our published literature [21, 22]. The P53-KO cells were constructed using the CRISPR/Cas9 technique from Syngentech Ltd. (Beijing, China). The construction details and constructive efficiency were presented in our previous report.

Prior to multi-omics detection, HBE cells were divided into two groups: the p53-wild type (p53-wt) and p53-KO groups. Cells in the two groups were harvested $24 \mathrm{~h}$ post $12.5 \mu \mathrm{g} / \mathrm{mL}$ silica treatment. Cells were shipped to 
OE Biotech Ltd. (Shanghai, China) for further transcriptomic and metabolite analyses. The detection steps of transcriptome sequencing were as follows: cellular total RNA was extracted; the TruSeq Stranded Total RNA LT kit was used to digest RasRNA; confirmed doublestranded cDNA through double-strand synergic reaction system; cDNA was purified; a tail was added to connect sequencing heads; PCR amplification was carried out to construct the RNA library. The GeneChip Fluidics Station 450, GeneChip Hybridization Oven 645, and GeneChip Scanner 3000 7G (Affymetrix, Santa Clara, CA, USA) were used to detect mRNA changes. Two values, fold change and $p$-value, were used to compare the differential expression status of the mRNAs in the two groups. Fold change was the ratio of the base mean in the $\mathrm{p}-53 \mathrm{KO}$ group to that in the p53-wt group. $\mathrm{p}<0.05$, and a fold change $>2$ were considered to indicate a significant differential expression [21]. Our previous study has reported the effects of a lncRNA-mRNA co-expression network on lung bronchial epithelial cells after radiation exposure [23].

For metabolomics detection, metabolites were extracted from the p53-wt and $\mathrm{p} 53-\mathrm{KO}$ groups after exposure to the same dose of silica and analyzed using gas chromatography-mass spectrometry (GC-MS) [24]. The cell treatments were the same as the ones used for transcriptomics. The GC-MS conditions were as follows: an Agilent $7890 \mathrm{~B}$ gas chromatography system coupled to an Agilent 5977A mass-selective detector (Agilent Technologies Inc., Santa Clara, CA). The column was an aDb- $5 \mathrm{~ms}$ capillary column $(30 \mathrm{~m} \times 0.25 \mathrm{~mm} \times 0.25$ $\mu \mathrm{m}$ : J\&W Scientific, Folsom, CA, USA). Carrier gas of high-purity helium with a purity of at least $99.999 \%$. The injection volume was $1 \mu \mathrm{L}$ with an ample inlet temperature of $260^{\circ} \mathrm{C}$. The initial column chamber temperature was $60^{\circ} \mathrm{C}$, rising to $125^{\circ} \mathrm{C}$ at a rate of $8{ }^{\circ} \mathrm{C} / \mathrm{min}$, and then to $210{ }^{\circ} \mathrm{C}$ at $5{ }^{\circ} \mathrm{C} / \mathrm{min}$, followed by heating to $270{ }^{\circ} \mathrm{C}$ at $10{ }^{\circ} \mathrm{C} / \mathrm{min}$ and maintaining at $305{ }^{\circ} \mathrm{C}$ for $5 \mathrm{~min}$ after increasing the rate of increase to $20^{\circ} \mathrm{C} / \mathrm{min}$. The full scan mode (SCAN) was used, with a scanning range of $\mathrm{m} / \mathrm{z}$ 50-500 [21]. To control the quality, the quality control samples were injected at regular intervals throughout the analytical run.

\section{Quantitative real-timepolymerase chain reaction (qRT-PCR)} For qRT-PCR detection, we first extracted RNA using TRIzol (Invitrogen, Carlsbad, CA, USA). Then, the ReverTra Ace qPCR RT Master Mix with gDNARemover (Toyobo, Osaka, Japan) was used to reverse-transcribec DNA based on the manufacturer's instructions. qRTPCR was carried out in our laboratory using the Super Real PreMix Plus (SYBR Green) kit (Tiangen Biotech, Beijing, China) on the CFX96TM Real-Time system
(Bio-Rad Laboratories, Hercules, CA, USA), to validate the changes and trends observed in the microarray analysis. The comparative $\mathrm{Ct}(2-\Delta \Delta \mathrm{Ct})$ method was used to calculate relative fold differences in mRNA expression between HBE p53-KOand HBE p53-wt cells. The calculated formula was $2^{-\Delta \Delta \mathrm{Ct}}=\Delta \mathrm{Ct}(\mathrm{p} 53-\mathrm{KO}) / \Delta \mathrm{Ct}(\mathrm{p} 53-\mathrm{wt})$ $\left(2^{-\Delta \Delta C t} \geq 2\right.$ indicated high expression).

\section{Bioinformatic and integrative analyses}

We performed bioinformatic analysis and integrative analysis on the results of transcriptomic and metabolomics profiling. Bioinformatic analysis included heat map construction, volcano map construction, GO (Gene Ontology) analysis, and KEGG (Kyoto Encyclopedia of Genes and Genomes) pathway analysis. GO analysis mainly comprises molecular functions, cellular components, and biological processes, which are available online http://geneontology.org/. The KEGG pathway analysis is a collection of manually-drawn pathway maps representing our knowledge of the molecular interaction, reaction, and relation networks for metabolism, genetic information processing, cellular processing, and so on available online https://www.kegg.jp/kegg/pathway.html. Integrated transcriptomic and metabolomic analyses were conducted using MetaboAnalyst (https://www. metaboanalyst.ca/MetaboAnalyst/home.xhtml). This software performed integrative analysis through a completely revamped web interface, with three new modules (MS Peaks to Pathways, Biomarker Meta-analysis, and Network Explorer), together with a companion R package (MetaboAnalystR) [25, 26]. Gene lists and metabolite lists were uploaded with fold changes $>2$ and $p<0.05$. The algorithm selection was a hypergeometric test, the topology measure was degree centrality, and the integration method combined queries.

\section{Cell experiments}

HBE cells were used to assess the IL4I1, p53, E-Cadherin mRNA levels changes following different metabolite treatments through qRT-PCR detection. Differential benzeneacetic acid treatments $(50 \mu \mathrm{M}, 100 \mu \mathrm{M}$, and $150 \mu \mathrm{M})$ and the effects on cell survival rate via the CCK- 8 method using the relative CCK- 8 assay kit, cell cycle changes after treatment with or without benzeneacetic acid and the biomarkers of epithelial-mesenchymal transition (EMT) changes with or without benzeneacetic acid treatment. These cell experiments were conducted to compare the differential changes and functions between p53-wt and p53-KO cells.

The CCK-8 assay was conducted as previously described [27]. Briefly, after adding $10 \mu \mathrm{L}$ CCK-8 reagent, the cells were mixed evenly and kept in an incubator for $1 \mathrm{~h}$. The absorbance (A) at $450 \mathrm{~nm}$ was measured using 
an enzyme-linked immunosorbent assay reader, and the growth curve was plotted. Three wells were set up in each group [27].

Then, flow cytometry was used for the detection of the cell cycle. The two groups of cells were fixed with $70 \%$ ethanol and then placed overnight in the refrigerator at $4{ }^{\circ} \mathrm{C}$. The cells were washed twice with pre-cooled PBS, and PI dyeing solution $(20 \times)$ and RNase A $(50 \times)$ were added to each well with cells. After incubation at $37^{\circ} \mathrm{C}$ in the dark for $0.5 \mathrm{~h}$, the cell cycle was detected using flow cytometry.

WB detection was conducted as previously reported $[22,28,29]$. In this study, antibodies against p53, E-cadherin, N-cadherin, and Vimentin were purchased from Cell Signaling Technology, Inc.(Danvers, MA, USA) and Abcam (Cambridge, UK). Images were captured and assessed using the ChemiDocXRS + system (Bio-Rad Laboratories). Protein expression was quantified using Image Lab software (Bio-Rad Laboratories). At least three independent replicates were analyzed for each sample.

\section{p53 research trends analysis}

To draw a global view of p53 research trends, we first used the keyword "p53"to search for p53-related funds over the past decade in the online National Natural Science Foundation of China (NSFC; http://www.nsfc.gov. $\mathrm{cn} /$ ), and the National Institute of Health in the USA (https://www.nih.gov/). We then used ArcGIS software to illustrate the funding number in the differential province in China. Then, p53-related publications over the decade were searched in the PubMed database, and the number of publications in China and the USA were also compared. Finally, we searched the p53-related invention patents from the Chinese, USA, and European patent databases in our university (Jove Eye; http://lib.jovee ye.com).

\section{Statistical method}

All data from the statistical analysis are presented as means \pm standard deviation (SD). The data were analyzed using SPSS 20.0, GraphPad Prism 8.0 (Graph-Pad Software Inc., San Diego, USA) and R 3.2.5 (DevelopmentCore Team, Vienna, Austria). The independent sample mean t-test was used for comparison between two groups. mRNA expression levels were compared using the two-tailed Student's t-test. $p<0.05$ indicated a significant difference.

\section{Results}

Deficiency of p53 significantly alters mRNA expression following silica stimulus

The verification of p53-knockout efficiency using CRISPR/Cas9 was examined with WB. The p53-knockout result has been reported in our previous study. In this study, we continued to ascertain whether p53 deficiency influenced the mRNA alterations following silica $(12.5 \mu \mathrm{g} / \mathrm{L})$ treatment in the p53-wt and p53-KO HBE cells. At $24 \mathrm{~h}$ post silica treatment, compared with the p53-wt group, mRNAs showed significant alterations in the $\mathrm{p} 53-\mathrm{KO}$ group. The expression levels of a total of 52 mRNAs changed significantly $(p<0.05)$, of which 31 mRNAs were upregulated and 21 were downregulated in the p53-KO group (Fig. 1a). We harvested cells for transcriptomic detection based on our findings from our pilot study that suggested that cell damage at this timepoint could significantly induce EMT [29-31]. The details of the changes in mRNA expression are listed in Table 1. It can be seen that ANKRD22, GALNT16, LGR5, ITGA9, and ELMOD1 were the five most strongly upregulated mRNAs, with fold changes of approximately $11,6,5.8,5.2$, and 5, respectively. Meanwhile, SLC9B1, GOLGA8Q, FAM19A5, LAIR2, and PLA2G2C were the five most strongly downregulated mRNAs, with downregulation fold changes of $0.05,0.06,0.06,0.07$, and 0.18 , respectively. Heatmap and volcano map assays further illustrated that compared with p53-wt cells, the number of the upregulated mRNAs was significantly higher than that of the downregulated mRNAs in the p53-KO cells (Fig. 1b-d). These results demonstrated that p53 deficiency led to changes in mRNA expression upon exposure to silica, suggesting that mRNA expression may be directly or indirectly regulated by $\mathrm{p} 53$.

To predict the differentially altered mRNA functions, we performed $\mathrm{GO}$ analysis on the top 30 differentially expressed mRNAs. As shown in Additional file 1: Fig. S1, the altered mRNAs were related to a large variety of cell structures and functions, including molecular junctions such as the nucleus, calcium ion binding, cellular components including the extracellular region and plasma membrane, biological processes including antimicrobial humoral response, and regulation of cell proliferation. To compare the signaling pathways involved between all differentially altered mRNAs $(p<0.05)$, we performed KEGG pathway analysis. The mRNAs with a significantly altered expression (Fig. 2a) were mainly involved in downstream signaling pathways, environmental adaption, nervous system, energy metabolism, lipid metabolism, glycan biosynthesis and metabolism, xenobiotics biodegradation, and metabolism. Furthermore, compared to the significantly downregulated mRNAs, the upregulated mRNAs were involved in the signaling pathway of the immune system, nucleotide metabolism, cancers, endocrine and metabolic diseases, and transport and catabolism. These data indicate that the deficiency of p53 significantly alters the expression of a large number of mRNAs and that these mRNAs may participate in 
Table 1 Top 20 differential expressed genes/mRNAs in the p53-wt vs. p53-KO HBE cells post $12.5 \mu \mathrm{g} / \mathrm{mL}$ silica

\begin{tabular}{|c|c|c|c|c|c|c|c|}
\hline \multicolumn{4}{|c|}{ Upregulated mRNA } & \multicolumn{4}{|c|}{ Downregulated mRNA } \\
\hline Gene-ID & Fold change & Log2 (Fold) & $p$ value & Gene-ID & Fold change & Log2 (Fold) & pvalue \\
\hline ANKRD22 & 11.33825 & 3.503126 & 0.031888 & SLC9B1 & 0.062922 & -3.99028 & 0.023555 \\
\hline GALNT16 & 5.9697 & 2.577658 & 0.015915 & GOLGA8Q & 0.067677 & -3.88519 & 0.040364 \\
\hline LGR5 & 5.805771 & 2.537488 & 0.00641 & FAM19A5 & 0.068239 & -3.87326 & 0.033308 \\
\hline ITGA9 & 5.201689 & 2.37898 & 0.033195 & LAIR2 & 0.07763 & -3.68725 & 0.035158 \\
\hline ELMOD1 & 5.045316 & 2.334945 & 0.038847 & PLA2G2C & 0.182084 & -2.45733 & 0.024876 \\
\hline GPT & 4.512969 & 2.174077 & 0.042918 & TNFRSF10C & 0.215358 & -2.21519 & 0.018619 \\
\hline SCG3 & 4.258003 & 2.090177 & 0.028393 & NEU4 & 0.227828 & -2.13399 & 0.040534 \\
\hline DEFA5 & 3.533064 & 1.82092 & 5.51E-05 & VAV1 & 0.325185 & -1.62067 & 0.002011 \\
\hline DEFA6 & 3.417341 & 1.772874 & 0.002371 & $\mathrm{SHC2}$ & 0.36299 & -1.462 & 0.044092 \\
\hline CRYBB2 & 3.367854 & 1.751829 & 0.022507 & LOC107985524 & 0.384776 & -1.37791 & 0.040135 \\
\hline JAKMIP2 & 3.267922 & 1.708373 & 0.022156 & $\mathrm{DLX} 2$ & 0.394143 & -1.34321 & 0.022467 \\
\hline ZNF385B & 3.169598 & 1.6643 & 0.018243 & FNTB & 0.437081 & -1.19403 & 0.039543 \\
\hline PRSS2 & 2.980291 & 1.575453 & 0.046668 & COL8A2 & 0.456718 & -1.13063 & 0.006895 \\
\hline RNF103-CHMP3 & 2.964665 & 1.567869 & 0.00646 & PSG8 & 0.458618 & -1.12464 & 0.015305 \\
\hline CHRNA1 & 2.764834 & 1.467193 & 0.009205 & SLC35F3 & 0.465274 & -1.10385 & 0.041656 \\
\hline LRRC46 & 2.747 & 1.457857 & 0.04034 & AS3MT & 0.466297 & -1.10068 & 0.006206 \\
\hline ADCY4 & 2.725217 & 1.446371 & 0.008368 & TREM2 & 0.47154 & -1.08455 & 0.000655 \\
\hline LIX1 & 2.71129 & 1.438979 & 0.041953 & KCNMA1 & 0.479557 & -1.06023 & 0.002141 \\
\hline KCND3 & 2.666753 & 1.415084 & 0.015511 & NANOS3 & 0.486488 & -1.03952 & 0.046132 \\
\hline SLC24A3 & 2.665332 & 1.414315 & 0.033314 & SLC45A1 & 0.491734 & -1.35907 & 0.008291 \\
\hline
\end{tabular}

$p<0.05$ represents significantly changed

cellular function in response to silica. Figure 2b, Additional file 2: Fig. S2 show the consistency of the microarray chip using five upregulated and five downregulated mRNAs. Our results were consistent with the microarray chip. This demonstrated that the microarray chip results can be used for downstream analyses and provide baseline information for cell experimentation.

\section{Deficiency of p53 significantly alters silica-mediated metabolites}

Metabolic adaption is an adaptive change occurring during the cells in response to silica under the p53-existing or deficiency status. The final goal of this adaptive change is to achieve a new homeostatic status and maximize the opportunity for cell survival [32]. In total, 42 metabolites changed significantly in $\mathrm{HBE}$ p53-KO cells compared with p53-wt cells $(p<0.05)$, of which 22 were upregulated and 20 were downregulated. Table 2 lists the 18 most altered metabolites. Among the top 18 differential metabolites, linoleic acid, butyric acid, alpha linolenic acid, stearic acid, and arachidonic acid were the five most strongly upregulated metabolites, with fold changes of approximately 91, 17, 16,13 , and 8, respectively. Oxoglutaric acid, beta_alanine, fumaric acid, 4_hydroxyphenylpyruvic acid, and
L_Thyronine were the five most strongly downregulated metabolites, with fold changes of approximately 0.05, $0.08,0.1,0.13$, and 0.16 , respectively. Metabolomics profiling demonstrated that p53 deficiency resulted in the dysregulation of multiple metabolites, which may result in changes in related metabolic pathways. Variable important in projection (VIP) analysis of significantly changed metabolites was conducted. The larger the VIP, the greater the contribution of this variable to the grouping. Using 1.00 as the VIP cutoff score, 18 upregulated and 18 downregulated metabolites were identified as being potentially closely associated with p53 deficiency, of which the six upregulated and six downregulated metabolites are presented in Fig. 3a, b.

The heatmap visually represents upregulated and downregulated metabolites. As shown in Fig. 4b, compared with the p53-wt group, metabolites were significantly upregulated in the p53 knockout group post-silica. KEGG enrichment pathway analysis showed that the significantly changed metabolites were enriched in phenylalanine metabolism, Glycine, serine, threonine, glutathione, porphyrin, chlorophyllglyoxylate, and dicarboxylate metabolism (Fig. 4b). Metabolomic data confirmed that $\mathrm{p} 53$ has a wide range of regulatory effects on intracellular metabolism via these metabolic pathways in response to silica. 


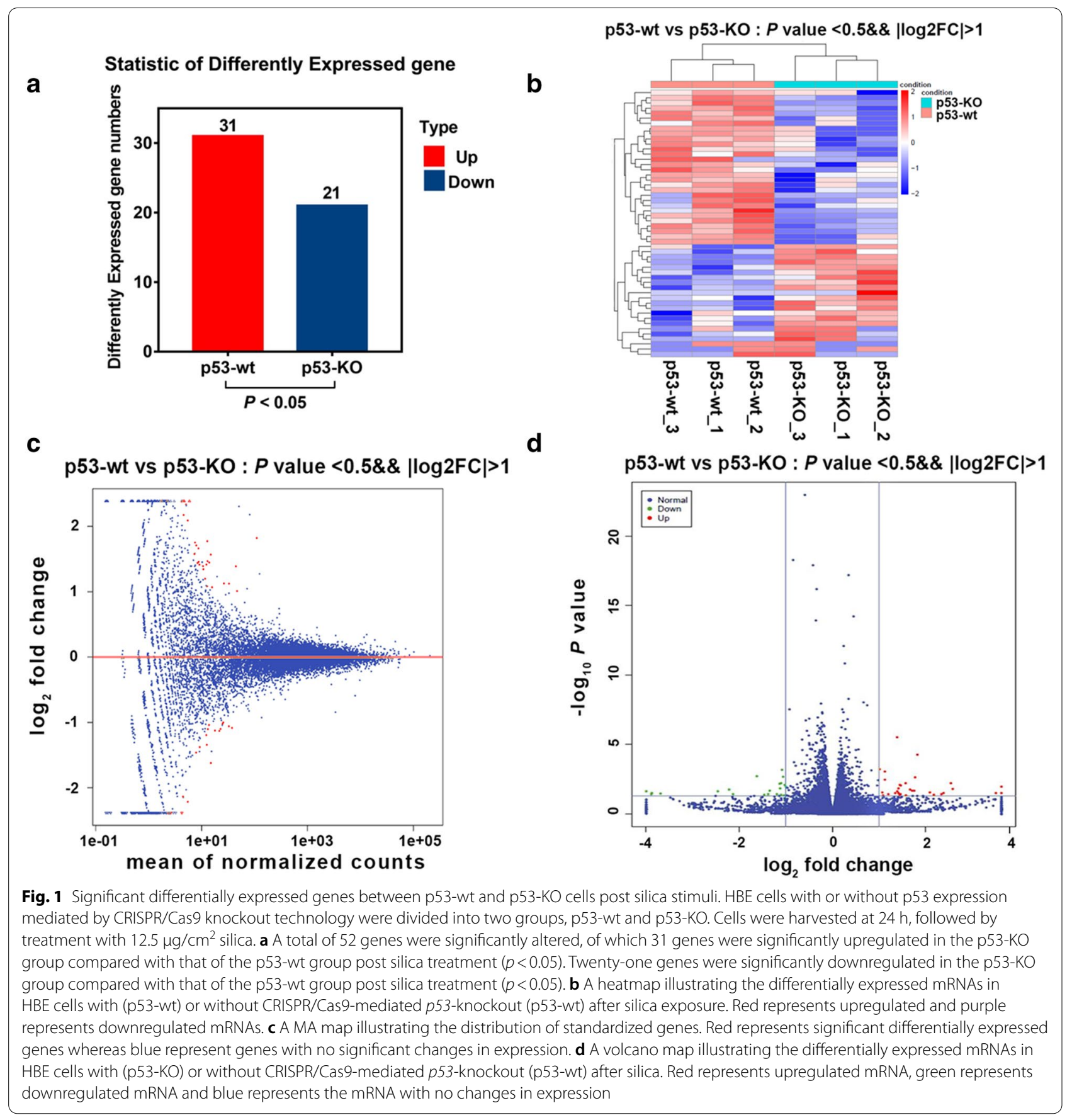

Integrated transcriptomic and metabolomic analyses of p53-dependent metabolic pathways

As our purpose of this study aimed at characterizing the transcriptomics and metabolomics changes occurring in $\mathrm{HBE}$ cells under silica exposure to compare p53 interactions and connections, we performed multi-omics analysis [33]. Notably, multi-omics can discover significantly perturbed pathways at both the metabolic and transcriptional levels, thereby providing a potential biomarker of silica-induced EMT and a basis for further investigation of the underlying molecular mechanisms [33]. Online MetaboAnalyst 4.0, using the PrimeFaces library (v7.0) based on the JavaServer Faces Technology, offering an efficient pipeline to support high-throughput global metabolomics in the opensource $\mathrm{R}$ environment [25] was utilized to perform our 


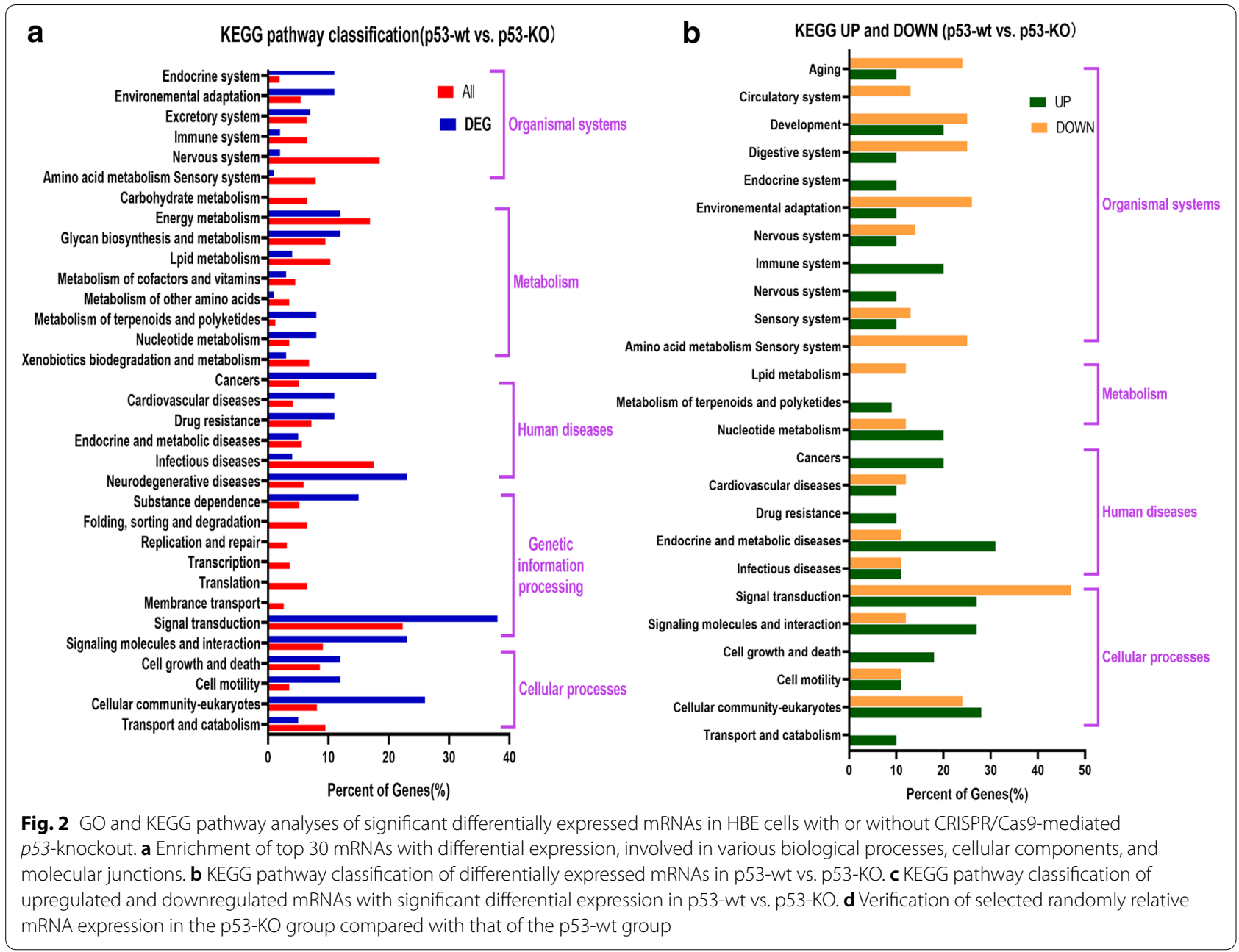

multi-omics in this study (https://www.metaboanalyst. $\mathrm{ca} /$ home.xhtml).

The mRNA enrichment and metabolic pathway analyses revealed 35 significantly altered pathways at both the metabolomic and mRNA expression levels in HBE p53-KO cells, where these pathways are involved in glyoxylate and dicarboxylate metabolism, citrate cycle (TCA), arginine biosynthesis, butanoate metabolism, alanine, aspartate, and glutamate metabolism, and phenylalanine metabolism (Table 3 ). Figure $5 \mathrm{~b}$ shows that there are four metabolic pathways, phenylalanine, glyoxylate, dicarboxylate, and linoleic acid metabolism and TCA had $p$-values $<0.05$ and impact coefficients $>0.2$, indicating that, in response to silica insult, these pathways may be dysregulated by p53. Figure $4 \mathrm{~d}$ illustrates the details of the phenylalanine metabolic pathway, indicating that phyenylpyruvate and phenyl-acetaldehyde levels and TAT expression were decreased and 2-hydroxy-3-phenylpropanoate and 2-hydroxy-phenylpropanoate levels were increased. Figure $5 \mathrm{a}$ illustrates the details of the TCA pathway, indicating that the citrate, cis-aconitate, 2-oxogluate, and succinate were decreased and oxaloacetate and oxalosuccinate levels were increased. Figure $5 \mathrm{~b}$ illustrates the details of the linoleic acid metabolic pathway, indicating that the linoleate was significantly increased, but PLA2G4B was decreased. These data indicate that the changes seen in mRNA expression levels may be related to p53-dependent alteration of metabolic pathways.

To validate the transcriptomic and metabolomic results, HBE cells were divided into four groups: p53-wt, p53-wt treated with $\mathrm{SiO}_{2}\left(\mathrm{p} 53-\mathrm{wt}-\mathrm{SiO}_{2}\right), \mathrm{p} 53-\mathrm{KO}$, and p53-KO cells treated with $\mathrm{SiO}_{2}\left(\mathrm{p} 53-\mathrm{KO}-\mathrm{SiO}_{2}\right)$. Cells were harvested $24 \mathrm{~h}$ after $12.5 \mu \mathrm{g} / \mathrm{L}$, and the IL4I1, HPD, ALDH3A1, ACLY, ACO, ALOX15, CYP2J2, and CYP1A2expression levels were determined using qRTPCR. As shown in Fig. 5c, compared with the untreated p53-wt group, theIL4I1, HPD, ALDH3A1, ACLY, and ACO expression levels were significantly increased, but the ALOX15, CYP2J2, and CYP1A2 expression levels were significantly reduced in $\mathrm{p} 53-\mathrm{wt}-\mathrm{SiO}_{2}$. On the other 
Table 2 Top 18 differential metabolites in the p53-wt vs. p53-KO HBE cells post $12.5 \mu \mathrm{g} / \mathrm{mL}$ silica

\begin{tabular}{|c|c|c|c|c|c|c|c|}
\hline \multicolumn{4}{|l|}{ Upregulated metabolites } & \multicolumn{4}{|l|}{ Downregulated metabolites } \\
\hline Gene-ID & Fold change & Log2 (Fold) & VIP score & Gene-ID & Fold change & Log2 (Fold) & VIP score \\
\hline Linoleic acid & 91.30556568 & 6.5126309 & 1.522815 & Oxoglutaric acid & 0.054212046 & -4.205242732 & 1.153971 \\
\hline Butyric acid & 17.21165834 & 4.105314202 & 1.239433 & Beta_Alanine & 0.083038327 & -3.590078819 & 1.266837 \\
\hline Alpha_Linolenic acid & 16.48137866 & 4.042765023 & 1.529557 & Fumaric acid & 0.106235462 & -3.234662673 & 1.066492 \\
\hline Stearic acid & 13.95122891 & 3.802320304 & 1.432926 & 4_Hydroxyphenylpyruvic acid & 0.138183866 & -2.855338915 & 1.262136 \\
\hline Arachidonic acid & 8.151991288 & 3.02715251 & 1.307153 & L_Thyronine & 0.160574413 & -2.638686077 & 1.33295 \\
\hline Docosahexaenoic acid DHA & 7.888603473 & 2.979769921 & 1.409017 & Pyroglutamic acid & 0.183156185 & -2.448853673 & 1.0547 \\
\hline Valeric acid & 6.513822845 & 2.703504484 & 1.326264 & P_Hydroxyphenylacetic acid & 0.18381705 & -2.443657506 & 1.293357 \\
\hline Formic acid & 5.821979245 & 2.541509696 & 1.244691 & Hydroxyphenyllactic acid & 0.188740975 & -2.405520436 & 1.228796 \\
\hline 11_cis_Eicosenoic acid & 4.970626572 & 2.313427722 & 1.428579 & 3_Nitrotyrosine & 0.214676537 & -2.219763575 & 1.294143 \\
\hline Indoleacrylic acid & 4.329224483 & 2.11410861 & 1.606161 & Benzeneacetic acid & 0.221848007 & -2.1723565 & 1.111947 \\
\hline Pentadecanoic acid & 3.848793752 & 1.944406363 & 1.180618 & Hydroxypropionic acid & 0.245830401 & -2.024264755 & 1.654862 \\
\hline Palmitic acid & 3.779422024 & 1.918165624 & 1.601808 & Glyceric acid & 0.258762543 & -1.950299297 & 1.025171 \\
\hline N_Phenylacetylphenylalanine & 3.737134456 & 1.90193247 & 1.476966 & L_Alanine & 0.259966065 & -1.943604783 & 1.098831 \\
\hline $\begin{array}{l}\text { 3,4_Dihydroxyhydrocinnamic } \\
\text { acid }\end{array}$ & 3.660604188 & 1.872081787 & 1.231095 & Cis and trans_Cinnamic acid & 0.271785878 & -1.879457598 & 1.155961 \\
\hline Docosapentaenoic acid 22n_6 & 2.176457553 & 1.121981884 & 1.026765 & Phenylacetic acid & 0.28363417 & -1.817896749 & 1.418577 \\
\hline Isobutyric acid & 1.771921197 & 0.825314444 & 1.012767 & Nicotinic acid & 0.284218303 & -1.814928632 & 1.497762 \\
\hline Heptadecanoic acid & 1.599045316 & 0.677210825 & 1.189061 & N_Acetylserotonin & 0.28649635 & -1.803411334 & 1.53686 \\
\hline Docosapentaenoic acid DPA & 1.480405616 & 0.565992513 & 1.072228 & Suberic acid & 0.303340107 & -1.720991836 & 1.305605 \\
\hline
\end{tabular}

VIP: Variable important in projection, The larger the VIP, the greater the contribution of this variable to the grouping; Log2 (FC): the ratio of the average expression amount of metabolites in the two groups of samples. A positive value indicates up regulation and a negative value indicates down regulation

hand, compared with untreated p53-KO cells, IL4I1, HPD, ALDH3A1, ACLY, and ACO expression levels were significantly reduced, whereas ALOX15, CYP2J2, and CYP1A2 expression levels were significantly reduced in the p53- $\mathrm{KO}-\mathrm{SiO}_{2}$ cells.

\section{Benzeneacetic acid is a p53-dependent metabolite that regulates EMT in HBE cells in response to silica}

Based on the integrative data provided above, we wondered whether there are key metabolites of p53-dependentinvolvement in the silica-induced EMT progression. We first conducted qRT-PCR to test several inflammation and EMT-related changes in genetic expression such as thatofIL4IL, p53, and E-cadherin treated with or without a few metabolites including benzeneacetic acid, linoleic acid, and arachidonic acid. As shown in Fig. 6a, compared with the DMSO control group, HBE cells treated with $100 \mu \mathrm{M}$ benzeneacetic acid showing significantly increased levels of p53 and E-Cadherin whereas linoleic acid and arachidonic acid had no significant effects on IL4IL, p53 and E-Cadherin expression. Additional file 3: Fig. S3A show the E-cadherin through immunofluorescence (IF) expression in HBE cells treated with silica, while Additional file 3: Fig. S3B show the actin fiber formation in HBE cells treated with silica. As shown in Fig. 6b, HBE cells treated with benzeneacetic acid for $48 \mathrm{~h}$ at indicated concentration showed no significant cell toxicity through CCK- 8 detection at the indicated doses, whereas in $\mathrm{HBE}$ p53-KO cells, benzeneacetic acid for $48 \mathrm{~h}$ at indicated concentration showed no significant cell toxicity (Fig. 6c). We also observed that p53-wt cells treated with benzeneacetic acid led to G1/S arrest, whereas in p53-KO cells, the G1/S transition was not affected by benzeneacetic acid treatment. This suggested that the G1/S arrest activation was p53-dependent (Fig. 6d, e). This result also is in line with previous study that benzeneacetic acid could be an inducer of G0/G1 arrest without significant cell toxicity [34-36]. Similarly, in p53-wt cells treated with benzeneacetic acid, p53 and E-Cadherin expression increased at the indicated time points, whereas in p53-KO cells, E-Cadherin expression was not significantly altered (Fig. 7a, b). Additional file 4: Fig. S4A-B shows the quantitative determination of relative p53 and E-cadherin expression in HBE p53-wt and HBE p53-KO cells post benzeneacetic acid exposure at the indicated time points. As of our previous published study have demonstrated that silica can induce EMT in HBE cells with p53 wild-type expression. Further, a deficiency of p53 led to decreased E-cadherin expression and increased $\mathrm{N}$-cadherin and $\alpha$-SMA expression in the HBE cells treated with silica, indicating the p53 plays critical role in the regulation of development of EMT [37]. 

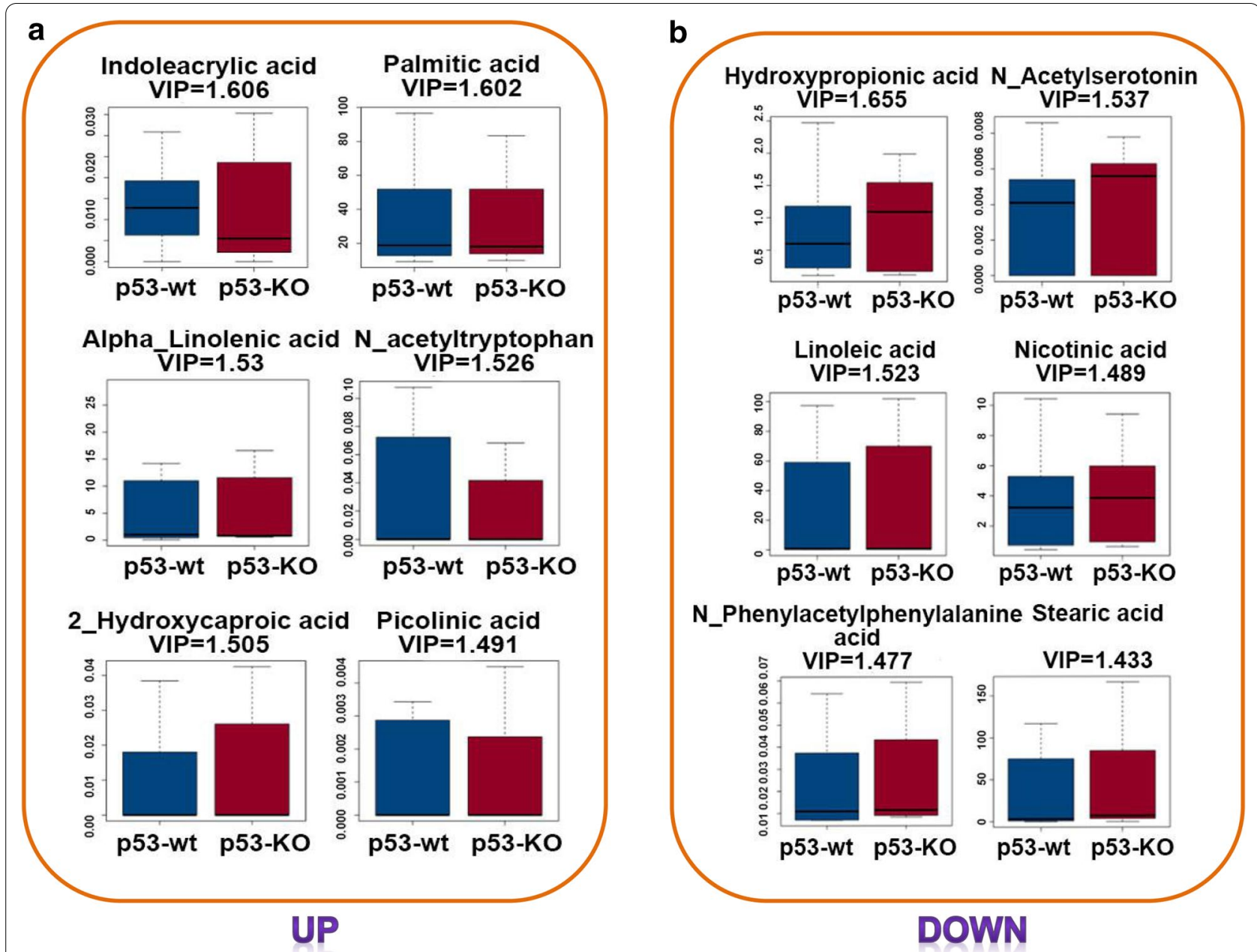

Fig. 3 Top six upregulated or downregulated metabolites in the p53-KO group compared with that of the p53-wt group post silica exposure. a Top six upregulated metabolites in the p53-KO group compared with that of the p53-wt group post silica exposure. b Top six downregulated metabolites in the p53-KO group compared with that of the p53-wt group post silica exposure. VIP means variable importance in the projection

Moreover, we performed other EMT biomarkers including N-cadherin, Vimentin, IL4I1 protein expression in HBE cells with or without p53 knockout post silica exposure (Additional file 5: Fig. S5).

We further used silica $\left(12.5 \mu \mathrm{g} / \mathrm{cm}^{2}\right)$ to treat p53-wt cells and detected changes in EMT biomarker proteins at indicated time points with or without intervention with benzeneacetic acid. The results showed that benzeneacetic acid increased p53 and E-Cadherin expression and decreased $\mathrm{N}$-cadherin and Vimentin expression (Fig. 7c), suggesting that intervention with benzeneacetic acid may attenuate the silica-induced EMT progression.

In summary, these results indicated that benzeneacetic acid may be a potential target for silica induced EMT. These findings are consistent with the transcriptomic and metabolomic results, providing further evidence that the benzeneacetic acid in the dysregulation of silica-related metabolic pathways may be p53-dependent. They also support the importance of p53 in the regulation of silicainduced cell EMT.

\section{p53-related research trends revealed the status of few transformations}

Since its discovery, p53 research-related publications have increased substantially. NSFC is the main source of investment for studies on p53 in China. As illustrated in Fig. 8a, from 2010 to 2019, 4225 projects were founded and the total amount of RMB allocated was approximately 1600 million. The funding amounts of the grants and numbers increased every year, with a slight decrease in 2016. The funded project content was mainly comprised of tumor etiology, digestive, respiratory, and urinary system tumors, and tumor recurrence and metastasis. Furthermore, to provide comparative information for the USA, we extracted p53-related research data from the NIH website. Between 1910 and 2019, the NIH 
a

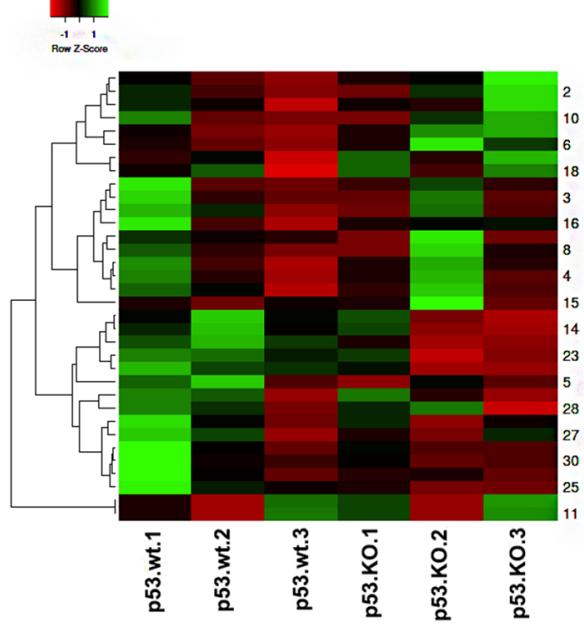

C

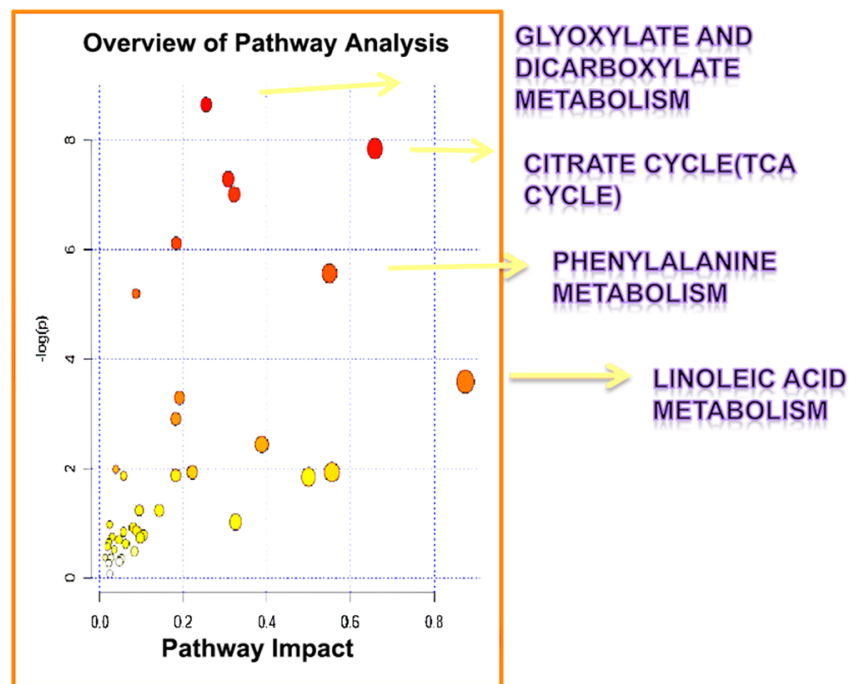

b

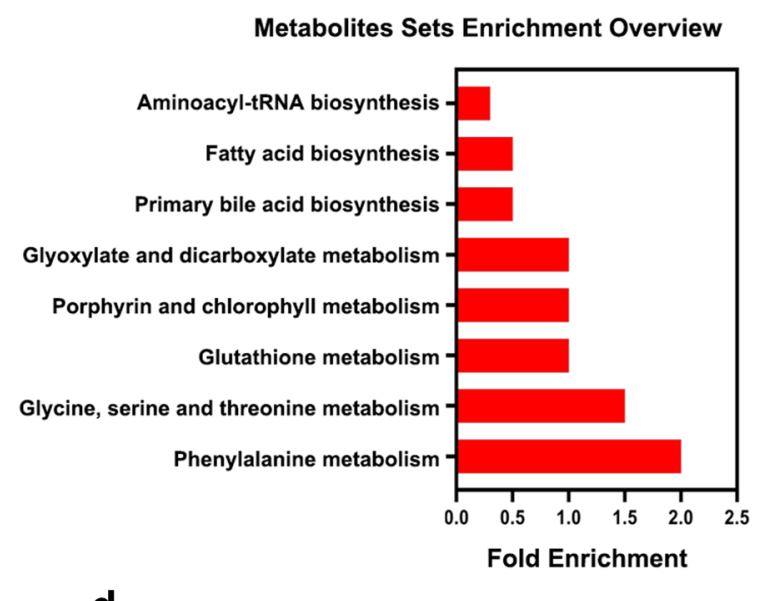

d

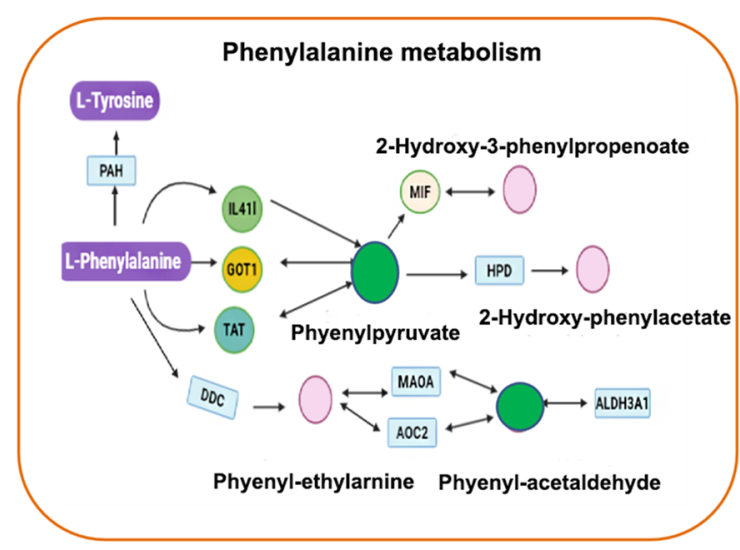

Fig. 4 Bioinformatic analysis of metabolomics. a A heatmap was used to visually represent upregulated and downregulated metabolites. b KEGG analysis illustrates the enrichment pathway for the significantly differential expressed metabolites. c Integrated transcriptomic and metabolomic analyses of p53-dependent metabolic pathways. d Details of phenylalanine metabolism. Pink represents slightly upregulated metabolites or genes, green represents significantly downregulated metabolites or genes, blue represents no significant changes

funded a total of approximately 10,800 projects relating to $\mathrm{p} 53$ research, with an approximate funding amount of USD 7200 million, substantially greater than that of the NSFC (Fig. 8b, c). To explore the institutions funded by NSFC, we used ArcGIS software to present the information visually. As shown in Fig. 8d, the funded projects were concentrated in developed areas or high-income provinces such as Beijing, Shanghai, and Guangdong Province.

As illustrated in Fig. 9a, from 2010 to 2019, the annual change was not large and remained stable in the 4000 to 5000 studies annually worldwide. However, compared with the USA, Chinese scholars have contributed increasingly to scientific findings. An interesting thing is that the number of publications from Chinese researchers has increased much more rapidly than that in the USA from 2017 onward, indicating that p53 research has been focused on in China (Fig. 9b). We further investigated the awarded invention patent information among China, America, and Europe. From 2011 to 2020, there are only approximately 30-50 annual awarded p53related invention patents in China. The number of US invention patents was between and 20-100 annually from 2011 to 2010, however, the numbers were greater in Europe compared with China and USA. This may be because Europe includes many developed countries such 
Table 3 Integrative metabolic pathways analysis on results obtained from combined metabolomics and gene expression

\begin{tabular}{|c|c|c|c|}
\hline Pathway name & Match status & $p$ & Impact \\
\hline Glyoxylate and dicarboxylate metabolism & $10 / 92$ & 0.000174 & 0.25455 \\
\hline Citrate cycle (TCA cycle) & $8 / 142$ & 0.000391 & 0.65854 \\
\hline Arginine biosynthesis & $6 / 76$ & 0.000679 & 0.30769 \\
\hline Butanoate metabolism & $6 / 77$ & 0.000899 & 0.32143 \\
\hline Alanine, aspartate and glutamate metabolism & $6 / 82$ & 0.002202 & 0.18333 \\
\hline Phenylalanine metabolism & $5 / 50$ & 0.003821 & 0.55 \\
\hline Biosynthesis of unsaturated fatty acids & $6 / 106$ & 0.005523 & 0.086957 \\
\hline Linoleic acid metabolism & $5 / 64$ & 0.027727 & 0.875 \\
\hline Propanoate metabolism & $6 / 115$ & 0.037239 & 0.19149 \\
\hline Glutathione metabolism & $5 / 70$ & 0.054837 & 0.18182 \\
\hline Glycine, serine and threonine metabolism & $4 / 44$ & 0.08738 & 0.38806 \\
\hline Fatty acid biosynthesis & $4 / 70$ & 0.13803 & 0.039062 \\
\hline D-Glutamine and D-glutamate metabolism & $5 / 132$ & 0.14551 & 0.22222 \\
\hline Synthesis and degradation of ketone bodies & $4 / 79$ & 0.14551 & 0.55556 \\
\hline Pyruvate metabolism & $4 / 92$ & 0.15422 & 0.18182 \\
\hline Tyrosine metabolism & $4 / 114$ & 0.15534 & 0.057471 \\
\hline Phenylalanine, tyrosine and tryptophan biosynthesis & $3 / 57$ & 0.15888 & 0.5 \\
\hline Alpha-Linolenic acid metabolism & $4 / 117$ & 0.29307 & 0.095238 \\
\hline Mucin type O-glycan biosynthesis & $3 / 74$ & 0.29307 & 0.14286 \\
\hline Arachidonic acid metabolism & $4 / 148$ & 0.36168 & 0.325 \\
\hline Tryptophan metabolism & $3 / 90$ & 0.37875 & 0.024096 \\
\hline Valine, leucine and isoleucine degradation & $2 / 33$ & 0.40123 & 0.08046 \\
\hline Glycerolipid metabolism & $3 / 94$ & 0.42493 & 0.088235 \\
\hline Terpenoid backbone biosynthesis & $3 / 94$ & 0.43402 & 0.057143 \\
\hline Ether lipid metabolism & $3 / 98$ & 0.46045 & 0.10526 \\
\hline Fatty acid degradation & $2 / 43$ & 0.47685 & 0.029703 \\
\hline Nicotinate and nicotinamide metabolism & $3 / 124$ & 0.48567 & 0.097561 \\
\hline Beta-Alanine metabolism & $2 / 69$ & 0.50185 & 0.046512 \\
\hline Pentose phosphate pathway & $2 / 73$ & 0.52518 & 0.021739 \\
\hline Lysine degradation & $1 / 12$ & 0.54015 & 0.0625 \\
\hline Porphyrin and chlorophyll metabolism & $2 / 79$ & 0.56871 & 0.019231 \\
\hline Sphingolipid metabolism & $2 / 89$ & 0.60199 & 0.035088 \\
\hline Glycolysis or Gluconeogenesis & $2 / 90$ & 0.62074 & 0.083333 \\
\hline Aminoacyl-tRNA biosynthesis & $3 / 194$ & 0.69254 & 0.013699 \\
\hline Fatty acid elongation & $2 / 98$ & 0.69748 & 0.027027 \\
\hline
\end{tabular}

as Germany. However, in these three regions, from 2017, the number of invention patents decreased significantly (Fig. 9c, d). The p53 research trends analysis revealed that although a large number of p53-related studies have been published, the translation from the bench to the bedside has been stagnant.

\section{Discussion}

Beyond p53 inactivation is essential for majority of human tumors, broading our view of p53 role in silicosis will extremely aid understanding as to how a disease caused by silica, the ubiquitous airborne contaminant can lead to
EMT progress. p53 is one of the most extensively studied cancer and environment stress-related adaptive proteins in the past four decades [38]. Strategies that target mutant p53 expression, focusing on degradation of mutated protein, restoring the wild type activity, forming p53 complexes with other proteins, or interfering with p53-related signaling pathways can potentially help prevent and control cancer [14, 38]. However, many related studies remain controversial. For instance, in lung cancer cells, p53 attenuated glucose uptake leading to glycosis inhibition, whereas, in muscle cells, p53 can induce glycolytic enzymes. The possible reason mainly depends on the research context 


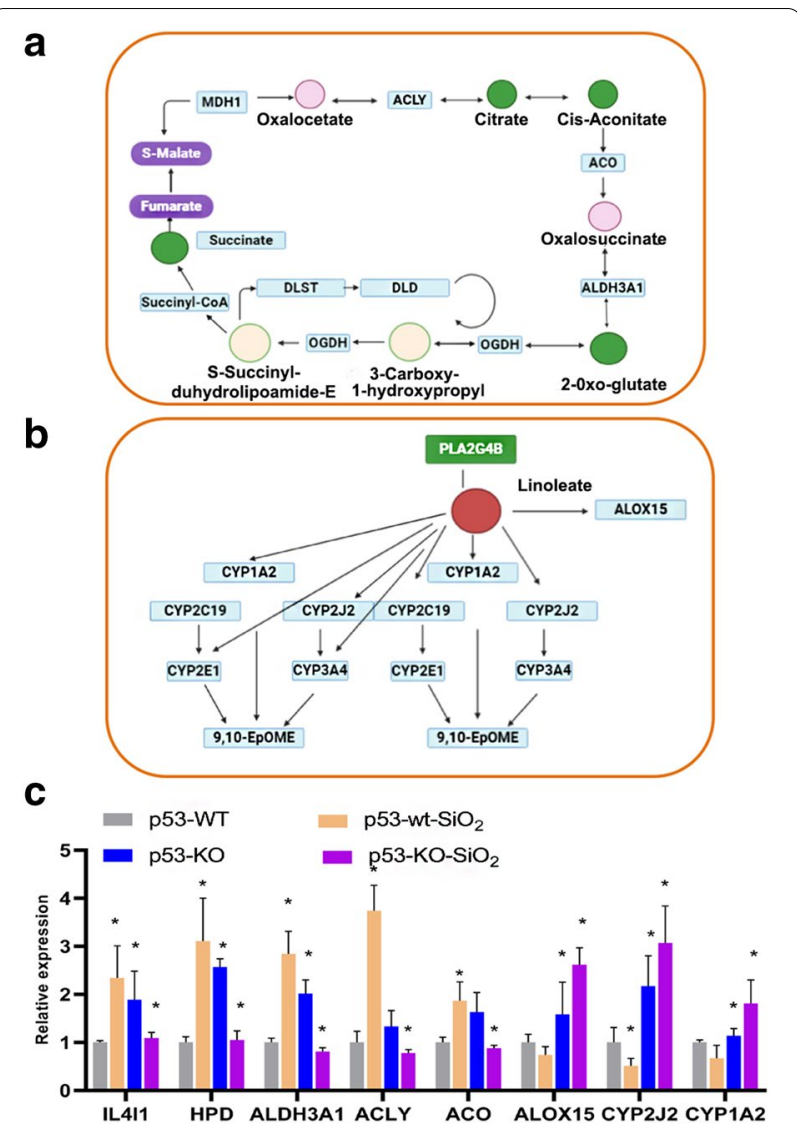

Fig. 5 Integrated transcriptomic and metabolomic analyses of p53-dependent metabolic pathways. a Details of the citrate cycle (TCA). Pink represents slightly upregulated metabolites or genes, green represents significantly downregulated metabolites or genes, blue represents insignificant changes. $\mathbf{b}$ Details of linoleic acid metabolism. Red represents significantly upregulated metabolites or genes, green represents significantly downregulated metabolites or genes, blue represents significant changes. c IL4I1, HPD, ALDH3A1, ACLY, ACO, ALOX15, CYP2J2, and CYP1A2 expression levels were determined using qRT-PCR in HBE cells of p53-wt, p53-wt treated with silica ( $\left.\mathrm{p} 53-\mathrm{wt}-\mathrm{SiO}_{2}\right), \mathrm{p} 53-\mathrm{KO}$, and $\mathrm{p} 53-\mathrm{KO}$ treated with silica ( $\mathrm{p} 53-\mathrm{KO}-\mathrm{SiO}_{2}$ ), respectively. Data are presented as means $\pm \mathrm{SD}$ from three independent experiments; ${ }^{*} p<0.05$ between different groups

which was pointed out by Edward et al. that the disparate p53activities and functions can be interpreted in different contexts [14]. Hence, in this study, we tried to knockout p53-wt from HBE cells and investigated the resulting interference in the downstream metabolic pathways. Our study revealed that following silica exposure resulted in HBE cell dysfunction through52 mRNAs that were putatively involved in p53-dependent pathways. Metabolomic profiling and bioinformatics analysis revealed that 42 metabolites were putatively involved in p53-dependent silica-mediated HBE cell dysfunction. Through integrated data analysis, we obtained three significant p53-dependent metabolic pathways, including phenylalanine, glyoxylate, dicarboxylate, and linoleic acid metabolism, and the TCA. In particular, we identified roles for benzeneacetic acid, a key regulation metabolite in the phenylalanine metabolic pathway, attenuated the silica-induced EMT in HBE cells in a p53-dependent manner.

Evidence shows that normal epithelial cells may be involved in controlling EMT in complicated mechanisms [39]. The classical function of $\mathrm{p} 53$ is to be a transcription cofactor for cells in response to various environmental stresses, including cell cycle arrest, apoptosis, and senescence [40]. However, recent findings suggest that p53 restricts epithelial cell plasticity [41]. This partly occurs due to the initiation of the p53-MDM2-SLUG pathway to enhance the epithelial biomarker E-Cadherin expression [42]. p53 also negatively regulates EMT progression by enhancing miR-34a expression to inhibit its downstream Snail1 expression, a mesenchymal marker that induces EMT progression [43]. Additionally, p53 is expressed in mesenchymal cells, indicating that there may be additional molecular mechanisms involved in the maintenance of epithelial integrity. In normal epithelial cells, p53 protein expression is augmented upon different stresses, including DNA damage and hyperproliferative signals [39]. p53 is also involved in the regulation of silica induced EMT progression. Cho et al. indicated that activated T $\beta R 1$ by silica induces the phosphorylation and degradation of RKIP, consequently resulting in Snail-mediated p53 suppression and the occurrence of EMT [44]. In this study, we compared the mRNA alterations and found that ANKRD22, GALNT16, LGR5, ITGA9, ELMOD1, SLC9B1, GOLGA8Q, FAM19A5, PLA2G2C, and LAIR2 were significantly changed post silica exposure when p53 was deficient. In p53-KO murine gastric epithelial (GIF-14) cells, EMT-induced plasticity is reflected in the expression of the embryonal proto-oncogene LGR5 [45], whereas the relationship between p53 and other mRNAs in EMT progression has not been reported. Further studies are warranted to confirm the alternations of these mRNAs in silica-induced p53 deficiency.

(See figure on next page.)

Fig. 6 Benzeneacetic acid attenuated silica-induced EMT progression in a p53-dependent manner. a Effects of benzeneacetic acid, linoleic acid, and arachidonic acid (concentration $=100 \mathrm{nM}$ ) on relative gene expression of IL4l1, p53, and E-Cadherin in HBE cells. $\mathbf{b}$ Effects of benzeneacetic acid on p53-wt HBE cell survival rate (\%) at indicated concentrations (50 nM, 100 nM, 150 nM). c Effects of benzeneacetic acid on p53-KO HBE cell survival rate (\%) at indicated concentrations $(50 \mathrm{nM}, 100 \mathrm{nM}, 150 \mathrm{nM}$ ). d Effects of benzeneacetic acid (concentration = $100 \mathrm{nM}$ ) on cell cycle in HBE cells. e Effects of benzeneacetic acid (concentration $=100 \mathrm{nM}$ ) on cell cycle in HBE cells with p53-KO 
a HBE

b

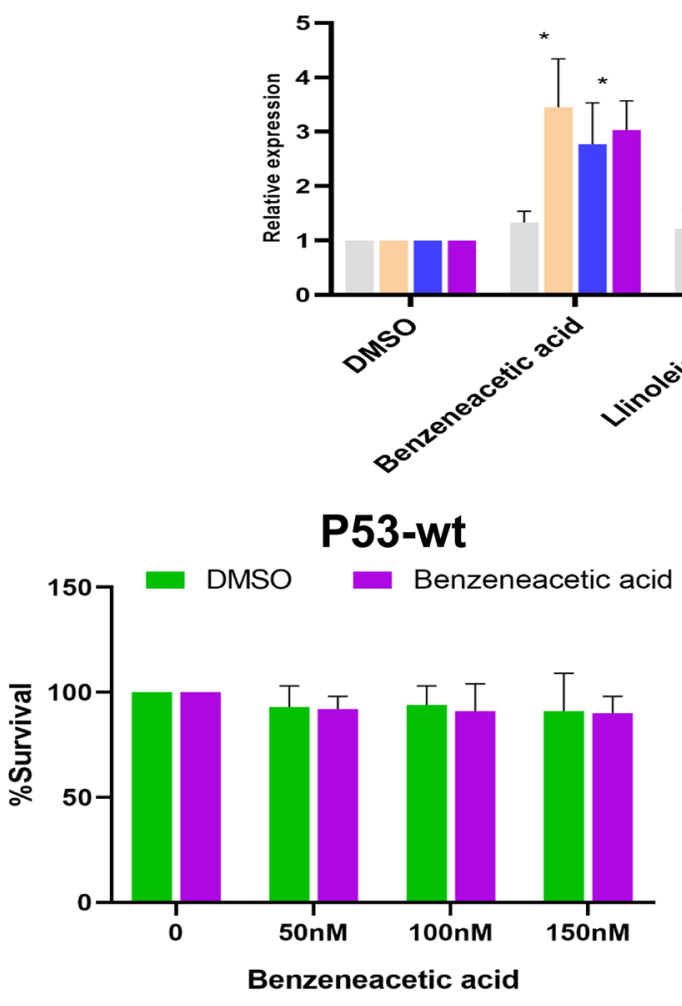

d
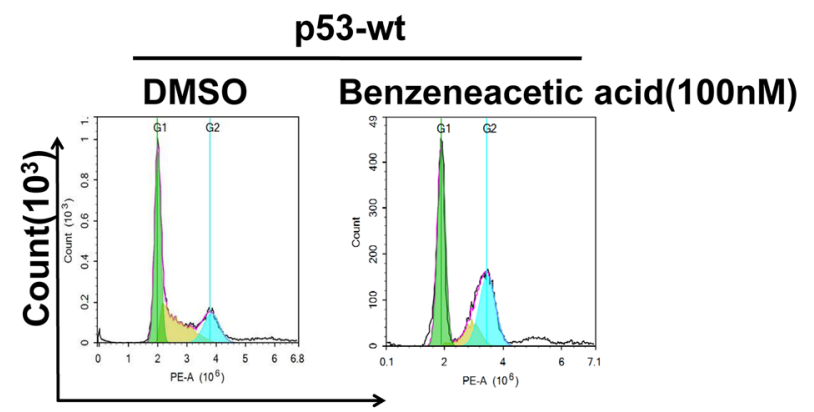

PE-A(106)

e
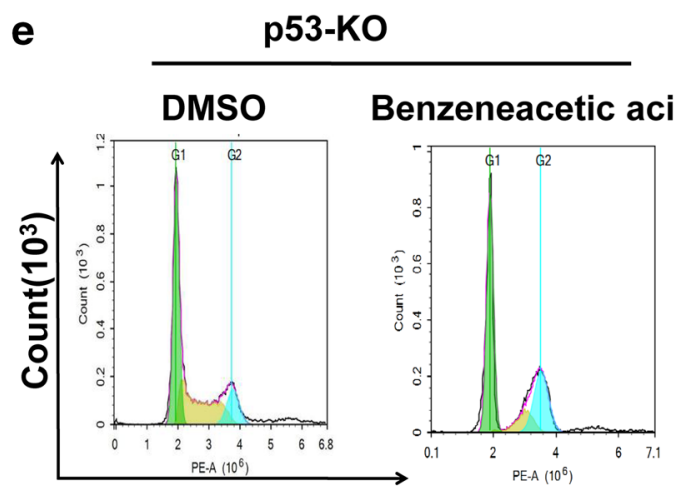

PE-A(106)
C

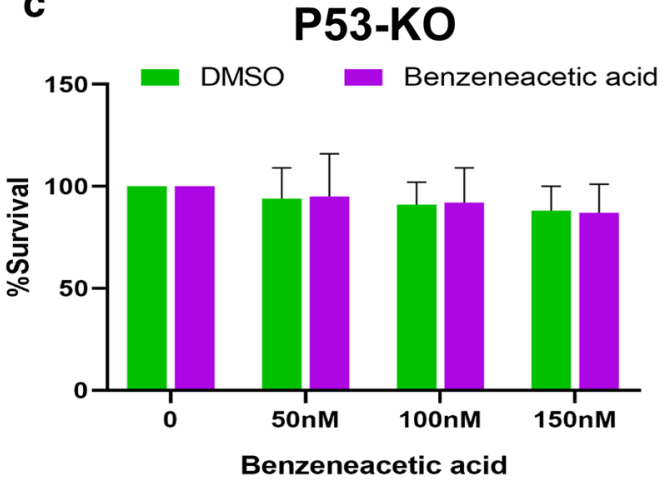

HBE

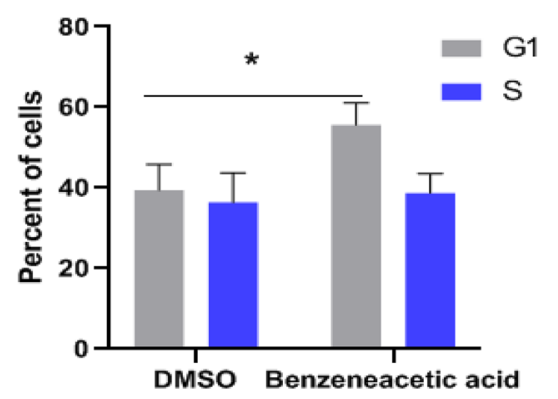

HBE

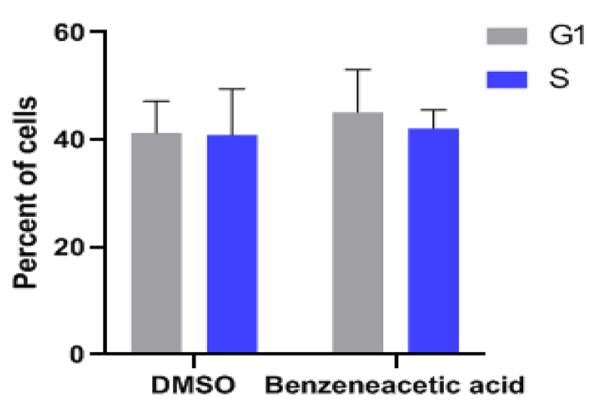




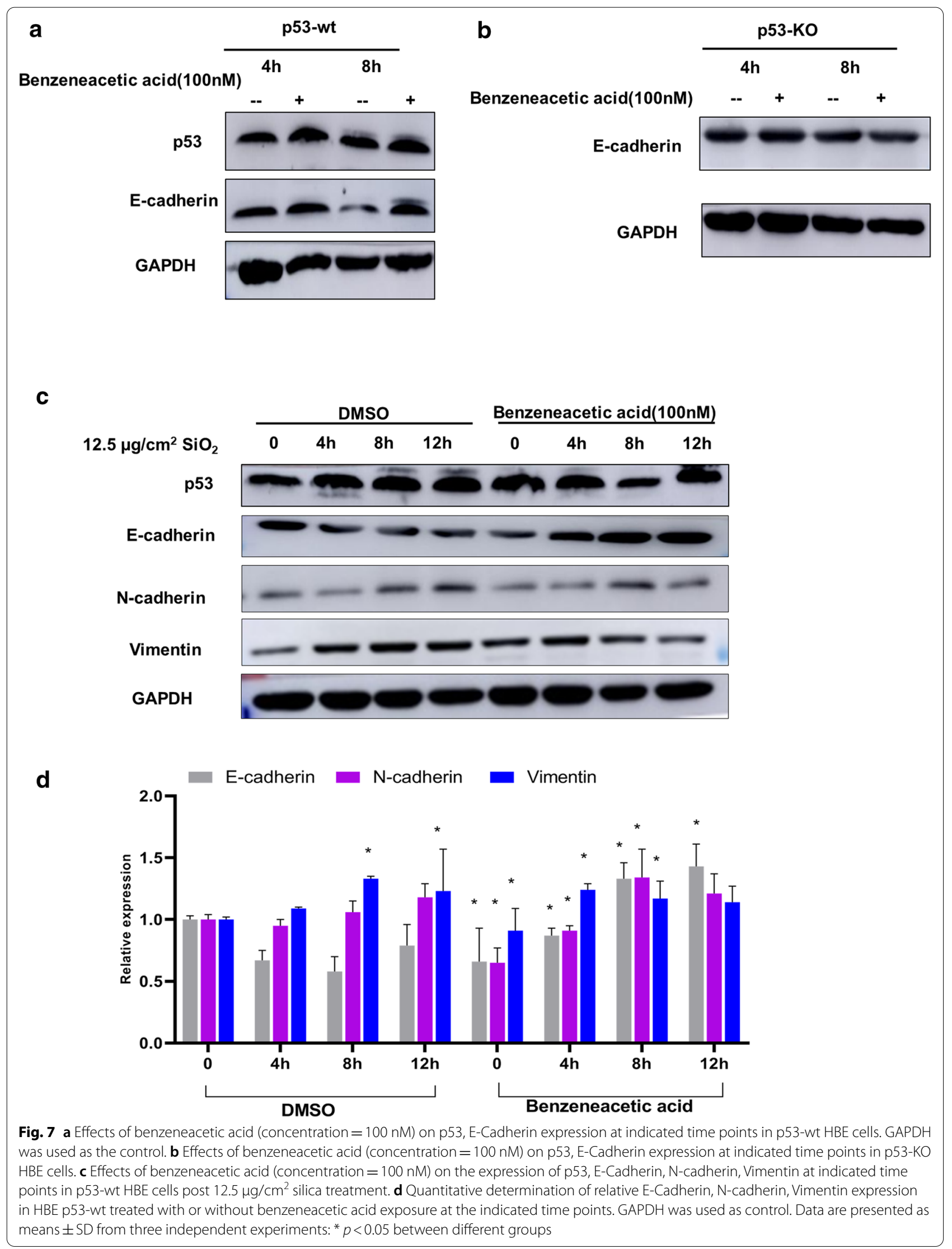




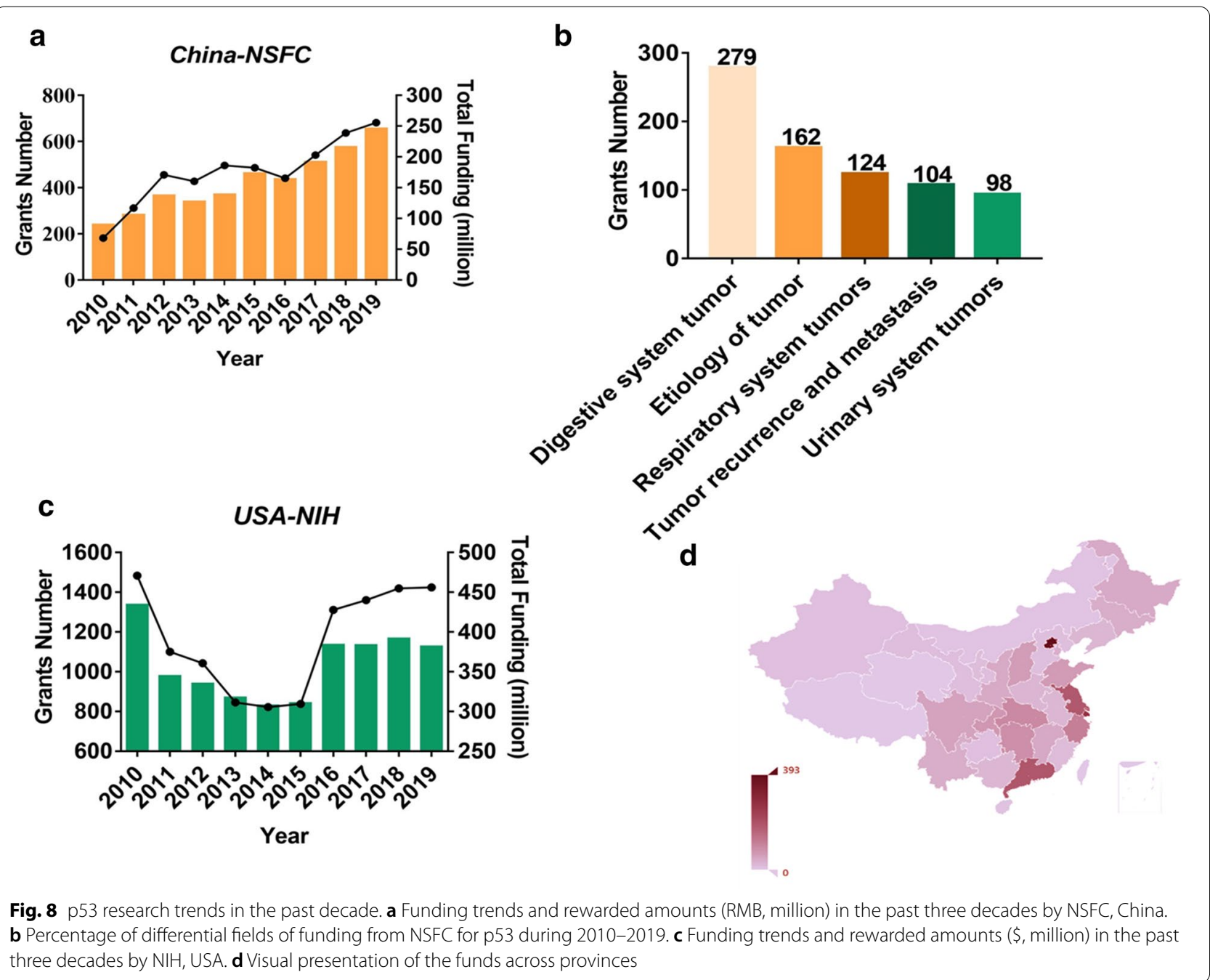

p53 regulation may be involved in EMT-related metabolic pathways. Sabine et al. suggested that treatment with calcitriol, the active vitamin D3 metabolite, activates p53 expression, and increases the repression of SNAIL, inhibiting EMT progression [46]. Fu et al. showed that restriction of phenylalanine modulates p53 and glucose metabolism in prostate cancer cells [47]. Karin et al. indicated that phenylalanine hydroxylase (PAH) is involved in the regulation of tyrosinase via p53 through the transcription of hepatocyte nuclear factor 1 alpha, which regulates melanogenesis [48]. Indeed, p53 controls multiple metabolic pathways, including glycolysis and pentose phosphate pathway, lipid, sphingolipid, amino acid, ammonia, and iron metabolism, mitochondrial biogenesis, integrity and respiration, TCA cycle, and ferroptosis [49] in normal cells, suggesting that deregulation of any associated metabolic activities contributes to several cancer and non-cancer human pathologies. Through this, it is obvious that p53 functions in metabolic pathways and displays certain cell and tissue specificity [49]. Thus, despite the classical function of the "guardian of the genome", p53 may be better characterized as a "guardian of homeostasis". Here, we report that phenylalanine, glyoxylate, dicarboxylate, and linoleic acid metabolism, and TCA may be involved in silica-stimuli in a p53-dependent manner. Phenylalanine metabolism belongs to amino acid metabolism. A recent study demonstrated that the retention of L-arginine, a semi exponential amino acid, increased p53 expression, and reduced the contents of pro-inflammatory cytokines in hepatic cells [50]. For TCA, the onco-metabolites stabilize the hypoxia inducible factor 1, inhibit p53 expression, and increase glutaminolysis, glycolysis, or dysregulation of EMT, suggesting that these changes in p53 and TCA-related metabolites are important driving forces of cancer pathogenesis and progression [51]. However, few studies have been reported on the p53-dependent changes in relative metabolites following silica exposure. We used 


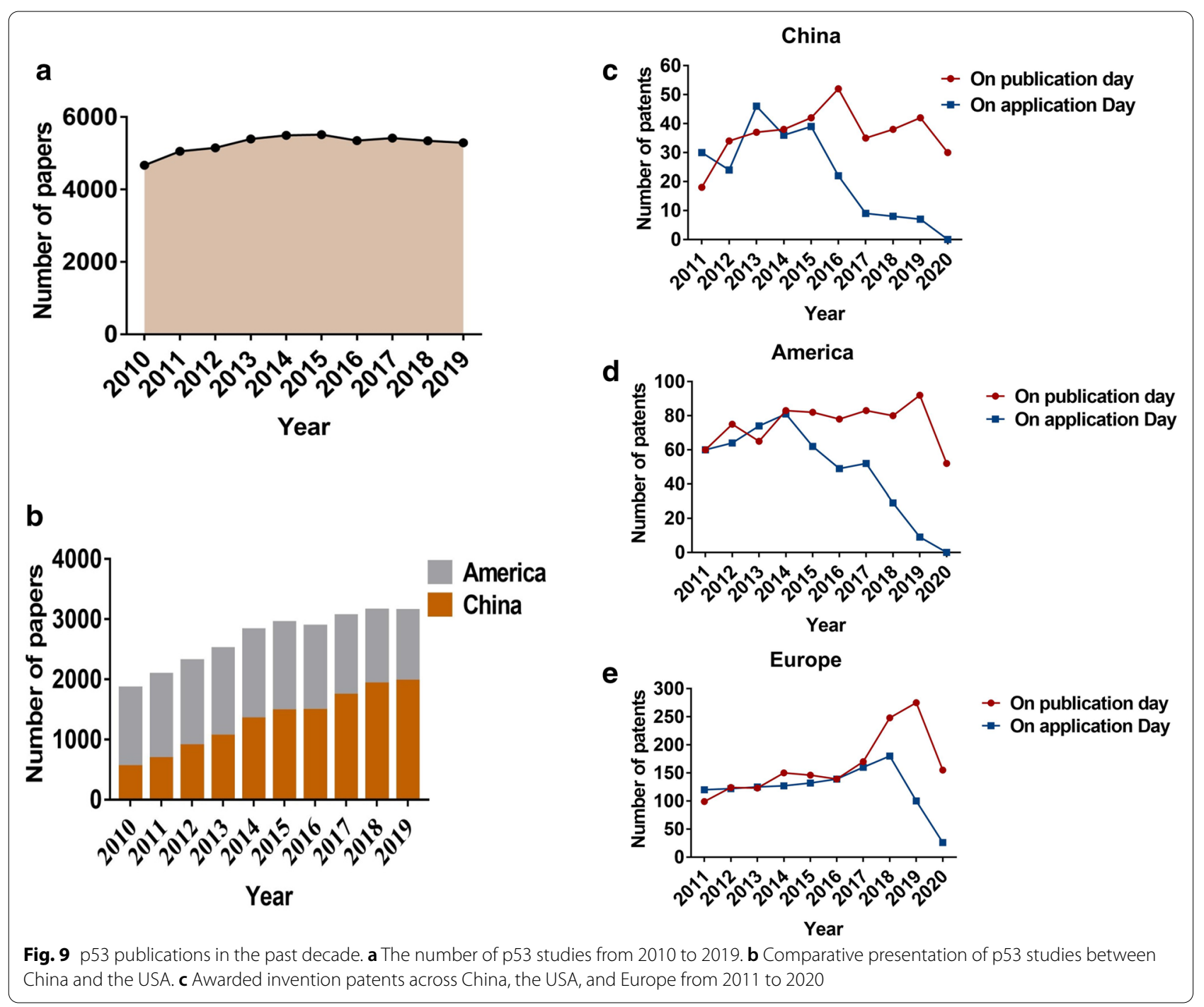

integrative multiple-omics analysis to provide baseline information for further p53 studies.

Benzeneacetic acid, was one of the major compounds among the 31 substances found in the ethyl acetate extract [52]. A previous study has shown that paraquat exposure in rats increases benzeneacetic acid levels [53]. Benzeneacetic acid has also been considered as an anti-inflammatory compound with the function of inhibiting lipopolysaccharide-induced inflammation in vivo [54]. Asynchronized smooth muscle cells treated with S-diclofenac (2-[(2,6-dichlorophenyl) amino] benzene acetic acid 4-(3H-1,2, dithiol-3-thione-5-yl) phenyl ester) showed an increase in stabilization of p53 [55]. In the KEGG database, benzeneacetic acid is involved in phenylalanine metabolism, and ALDH3, feaB, and paaI are thought to promote its levels, thereby increasing phenyl-acetylglutamine. Despite these reports, a mechanistic link between benzeneacetic acid and silica induced EMT has not yet been reported. The present study confirms the major role of p53-dependent benzeneacetic acid generation in normal HBEs post the silica stimulus. Notably, increased benzeneacetic acid levels in p53-wt cells inhibited the silica-induced EMT progression. The present study suggests that certain clinical benefits of benzeneacetic acid observed with p53 existence status may be in part mediated via attenuation in silica-triggered EMT signaling events in vitro. Although the present study focuses only on the function of benzeneacetic acid in human HBE cells, one can speculate that benzeneacetic acid has the potential to modulate other EMT-related markers or pathways, and accordingly, may potentially influence multiple silica-linked physiological processes.

Research trends regarding p53 research and control provide a closer look at this field. Since its discovery 40 years ago, p53 remains heavily investigated in the 
global scientific community, specifically in the USA. China has rapidly advanced the understanding of p53 in recent years, with large government investments through NSFC foundations. However, the clinical translation of p53 research is still challenging, despite a few studies that have applied for invention patents. This reflects the lack of innovative research and innovation. Olivier et al. emphasized that p53 translational research for improving cancer detection, prognosis, prevention, and therapy based on p53 study is critical in qualitative terms. p53 research has been conducted for three periods: the discovery from 1980 to 1990, first considered an oncogene factor; the rapid development study period from 1990 to 2010, second mainly characterized as multi-functional (transcription factor and causing double-edge effects in the various contexts); the third period was from 2010 to present, where research lacks substantial findings and clinical translation has been stagnating. At this time, we should encourage and incentivize high-impact metabolic future studies that use rare human disease contexts and novel animal models that can be clinically translatable.

Although p53 research has been intensive and obtained extremely discoveries, there still remains much to explore for p53 origin and regulation. Challenge in the coming era of p53 research would be the first of the comprehensive p53 function in other diseases instead of cancers, the second is the urgent need to translate p53 research results into clinical application. And new technologies applied in p53 function explore is also a big challenge for more robust clinical advances.

\section{Supplementary Information}

The online version contains supplementary material available at https://doi. org/10.1186/s13578-021-00545-0.

\section{Acknowledgements}

Not applicable.

\section{Authors' contributions}

RXH contributed to the study concept and critical design of the study. PD, JJ, and HJP conducted data analysis, MLZ, JZ, JJ conducted the experiments. RXH analyzed and interpreted the data. RXH wrote the initial manuscript, critically reviewed and revised the final manuscript. All authors read and approved the final manuscript.

\section{Funding}

This study was supported by grants from the National Natural Science Foundation of China (Grant No. U1803124 and 82073486).

\section{Availability of supporting data \\ Not applicable.}

Ethics approval and consent to participate

Not applicable.

\section{Consent for publication}

Not applicable.
Competing interests

The authors declare no competing financial interests.

\section{Author details}

${ }^{1}$ Department of Occupational and Environmental Health, Xiangya School of Public Health, Central South University, Changsha 410078, Hunan, China.

${ }^{2}$ Zhejiang Provincial Center for Cardiovascular Disease Prevention and Control,

Zhejiang Hospital, Hangzhou, Zhejiang, China.

Received: 11 October 2020 Accepted: 22 January 2021

Published online: 05 February 2021

\section{References}

1. Brey C, et al. Lung cancer related to occupational exposure: an integrative review. Revista gaucha de enfermagem. 2020;41:e20190378. https://doi. org/10.1590/1983-1447.2020.20190378.

2. Vis B, Powell JJ, Hewitt RE. Imaging flow cytometry methods for quantitative analysis of label-free crystalline silica particle interactions with immune cells. AIMS Biophys. 2020;7:144-66. https://doi.org/10.3934/ biophy.2020012.

3. Barbieri PG, Somigliana A, Carradori G. Severe silicosis due to diatomaceous earth in dental alginate: a necropsy study. La Medicina del Lavoro. 2020;111:222-31. https://doi.org/10.23749/mdl.v111i3.9742.

4. Carrieri M, Guzzardo C, Farcas D, Cena LG. Characterization of silica exposure during manufacturing of artificial stone countertops. Int J Environ Res Public Health. 2020;17:4489. https://doi.org/10.3390/ijerph17124489.

5. Ulvestad B, et al. Pulmonary function and high-resolution computed tomography in outdoor rock drillers exposed to crystalline silica. Occup Environ Med. 2020. doi:https://doi.org/10.1136/oemed-2019-106254.

6. Leon-Jimenez A, et al. Artificial stone silicosis: rapid progression following exposure cessation. Chest. 2020. https://doi.org/10.1016/j.chest .2020.03.026.

7. Kirby T. Australia reports on audit of silicosis for stonecutters. Lancet. 2019;393:861. doi:https://doi.org/10.1016/S0140-6736(19)30478-7.

8. Bell JL, Mazurek JM. Trends in pneumoconiosis deaths-United States, 1999-2018. MMWR Morb Mortal Wkly Rep. 2020;69:693-8. https://doi. org/10.15585/mmwr.mm6923a1.

9. Schmaling K, Kaplan RM, Porzsolt F. Efficacy and effectiveness studies of depression are not well-differentiated in the literature: a systematic review. BMJ Evid Based Med. 2020. https://doi.org/10.1136/bmjeb m-2020-111337.

10. Ning $\mathrm{H}$, et al. Challenges to improving occupational health in China. Occup Environ Med. 2017;74:924-5. doi:https://doi.org/10.1136/oemed $-2017-104656$

11. Barnes H, Goh NSL, Leong TL, Hoy R. Silica-associated lung disease: an old-world exposure in modern industries. Respirology. 2019;24:1165-75. https://doi.org/10.1111/resp.13695.

12. Cavalin C, et al. Beyond silicosis, is the world failing on silica hazards? Lancet Respir Med. 2019;7:649-50. https://doi.org/10.1016/S2213 $-2600(19) 30174-2$.

13. Lane DP, Crawford L. V.T antigen is bound to a host protein in SV40-transformed cells. Nature. 1979;278:261-3. doi:https://doi.org/10.1038/27826 $1 \mathrm{a} 0$.

14. Kastenhuber ER, Lowe SW. Putting p53 in context. Cell. 2017;170:1062-78. https://doi.org/10.1016/j.cell.2017.08.028.

15. Madenspacher $\mathrm{JH}$, et al. p53 Integrates host defense and cell fate during bacterial pneumonia. J Exp Med. 2013;210:891-904. https://doi. org/10.1084/jem.20121674.

16. Cheng Y, et al. CircRNA-012091/PPP1R13B-mediated lung fibrotic response in silicosis via endoplasmic reticulum stress and autophagy. Am J Respir Cell Mol Biol. 2019;61:380-91. https://doi.org/10.1165/rcmb.2019O017OC.

17. Wang W, et al. p53/PUMA expression in human pulmonary fibroblasts mediates cell activation and migration in silicosis. Sci Rep. 2015;5:16900. https://doi.org/10.1038/srep16900.

18. Wang $L$, et al. Essential role of p53 in silica-induced apoptosis. Am J Physiol Lung Cell Mol Physiol. 2005;288:L488-96. doi:https://doi.org/10.1152/ ajplung.00123.2003. 
19. Wang Z, et al. 20(S)-Protopanaxatriol promotes the binding of P53 and DNA to regulate the antitumor network via multiomic analysis. Acta Pharm Sin B. 2020;10:1020-35. https://doi.org/10.1016/j.apsb.2020.01.017.

20. Huang J, et al. Integrative omics analysis of p53-dependent regulation of metabolism. FEBS Lett. 2018:592:380-93. doi:https://doi. org/10.1002/1873-3468.12968.

21. Huang R, Liu X, Li H, Zhou Y, Zhou PK. Integrated analysis of transcriptomic and metabolomic profiling reveal the p53 associated pathways underlying the response to ionizing radiation in HBE cells. Cell Biosci. 2020;10:56. doi:https://doi.org/10.1186/s13578-020-00417-z.

22. Zhang J, Ding L, Sun G, Ning H, Huang R. Suppression of LINC00460 mediated the sensitization of HCT1 16 cells to ionizing radiation by inhibiting epithelial-mesenchymal transition. Toxicol Res (Camb). 2020;9:10716. doi:https://doi.org/10.1093/toxres/tfaa010.

23. Liu XD, et al. Integrated analysis of IncRNA-mRNA co-expression networks in the alpha-particle induced carcinogenesis of human branchial epithelial cells. Int J Radiat Biol. 2019;95:144-55. doi:https://doi. org/10.1080/09553002.2019.1539880.

24. Zhang $\mathrm{H}$, et al. Metabolomics reveals how cucumber (Cucumis sativus) reprograms metabolites to cope with silver ions and silver nanoparticleinduced oxidative stress. Environ Sci Technol. 2018;52:8016-26. https:// doi.org/10.1021/acs.est.8b02440.

25. Pang Z, Chong J, Li S, Xia J. MetaboAnalystR 3.0: toward an optimized workflow for global metabolomics. Metabolites. 2020;10:186. https://doi. org/10.3390/metabo10050186.

26. Chong J, Xia J. Using MetaboAnalyst 4.0 for metabolomics data analysis, interpretation, and integration with other omics data. Methods Mol Biol. 2020;2104:337-60. https://doi.org/10.1007/978-1-0716-0239-3_17.

27. Liu W, Huang M, Zou Q, Lin W. Curcumin suppresses gastric cancer biological activity by regulation of miRNA-21: an in vitro study. Int J Clin Exp Pathol. 2018;11:5820-9.

28. Mo LJ, et al. Exosome-packaged miR-1246 contributes to bystander DNA damage by targeting LIG4. Br J Cancer. 2018;119:492-502. doi:https://doi. org/10.1038/s41416-018-0192-9.

29. Huang R, Liu X, Li H, Ning H, Zhou PK. PRKCSH alternative splicing involves in silica-induced expression of epithelial-mesenchymal transition markers and cell proliferation. Dose Response. 2020;18:1559325820923825. https://doi.org/10.1177/1559325820923825.

30. Feng $F$, et al. Tanshinone IIA attenuates silica-induced pulmonary fibrosis via Nrf2-mediated inhibition of EMT and TGF-beta1/Smad signaling. Chem Biol Interact. 2020;319:109024. doi:https://doi.org/10.1016/j. cbi.2020.109024.

31. Zhou Y, Chen L, Sun G, Li Y, Huang R. Alterations in the gut microbiota of patients with silica-induced pulmonary fibrosis. J Occup Med Toxicol. 2019;14:5. https://doi.org/10.1186/s12995-019-0225-1.

32. Fernandez-Calero T, et al. Fine-tuning the metabolic rewiring and adaptation of translational machinery during an epithelial-mesenchymal transition in breast cancer cells. Cancer Metab. 2020;8:8. doi:https://doi. org/10.1186/s40170-020-00216-7.

33. Su P, et al. Integrated metabolo-transcriptomics and functional characterization reveals that the wheat auxin receptor TIR1 negatively regulates defense against Fusarium graminearum. J Integr Plant Biol. 2020. doi:https://doi.org/10.1111/jipb.12992.

34. Onishi T, Yamakawa K, Franco OE, Suzuki R, Kawamura J. p27Kip1 is the key mediator of phenylacetate induced cell cycle arrest in human prostate cancer cells. Anticancer Res. 2000;20:3075-81.

35. Stockhammer $\mathrm{G}$, et al. Inhibition of proliferation and induction of differentiation in medulloblastoma- and astrocytoma-derived cell lines with phenylacetate. J Neurosurg. 1995;83:672-81. doi:https://doi.org/10.3171/ jns.1995.83.4.0672.

36. Samid D, Shack S, Sherman LT. Phenylacetate: a novel nontoxic inducer of tumor cell differentiation. Cancer Res. 1992;52:1988-92.

37. Huang $R$, et al. The $p 53 / R M R P / m i R 122$ signaling loop promotes epithelialmesenchymal transition during the development of silica-induced lung fibrosis by activating the notch pathway. Chemosphere. 2021;263:128133. doi:https://doi.org/10.1016/j.chemosphere.2020.128133.

38. Muller PA, Vousden KH. Mutant p53 in cancer: new functions and therapeutic opportunities. Cancer Cell. 2014;25:304-17. doi:https://doi. org/10.1016/j.ccr.2014.01.021.
39. Oikawa T, Otsuka Y, Sabe H. p53-dependent and -independent epithelial integrity: beyond miRNAs and metabolic fluctuations. Cancers. 2018. https://doi.org/10.3390/cancers10060162.

40. Bieging KT, Mello SS, Attardi LD. Unravelling mechanisms of p53-mediated tumour suppression. Nat Rev Cancer. 2014;14:359-70. doi:https:// doi.org/10.1038/nrc3711.

41. Rinon A, et al. p53 coordinates cranial neural crest cell growth and epithelial-mesenchymal transition/delamination processes. Development. 2011;138:1827-38. doi:https://doi.org/10.1242/dev.053645.

42. Wang SP, et al. p53 controls cancer cell invasion by inducing the MDM2mediated degradation of Slug. Nat Cell Biol. 2009;11:694-704. doi:https:// doi.org/10.1038/ncb1875.

43. Kim NH, et al. A p53/miRNA-34 axis regulates Snail1-dependent cancer cell epithelial-mesenchymal transition. J Cell Biol. 2011;195:417-33. doi:https://doi.org/10.1083/jcb.201103097.

44. Cho JH, et al. Loss of NF2 induces TGFbeta receptor 1-mediated noncanonical and oncogenic TGFbeta signaling: implication of the therapeutic effect of TGFbeta receptor 1 inhibitor on NF2 syndrome. Mol Cancer Ther. 2018;17:2271-84. https://doi.org/10.1158/1535-7163.MCT-17-1210.

45. Voon DC, et al. EMT-induced stemness and tumorigenicity are fueled by the EGFR/Ras pathway. PLoS ONE. 2013;8:e70427. https://doi. org/10.1371/journal.pone.0070427.

46. Hunten $\mathrm{S}$, Hermeking $\mathrm{H}$. p53 directly activates cystatin D/CST5 to mediate mesenchymal-epithelial transition: a possible link to tumor suppression by vitamin D3. Oncotarget. 2015;6:15842-56. https://doi. org/10.18632/oncotarget.4683.

47. Fu YM, Lin H, Liu X, Fang W, Meadows GG. Cell death of prostate cancer cells by specific amino acid restriction depends on alterations of glucose metabolism. J Cell Physiol. 2010;224:491-500. https://doi.org/10.1002/ jcp.22148.

48. Schallreuter KU, Kothari S, Chavan B, Spencer JD. Regulation of melanogenesis-controversies and new concepts. Exp Dermatol. 2008;17:395404. doi:https://doi.org/10.1111/j.1600-0625.2007.00675.x.

49. Lacroix M, Riscal R, Arena G, Linares LK, Le Cam L. Metabolic functions of the tumor suppressor p53: implications in normal physiology, metabolic disorders, and cancer. Mol Metab. 2020;33:2-22. https://doi.org/10.1016/j. molmet.2019.10.002.

50. Zhang H, et al. Dietary supplementation of L-arginine and N-carbamylglutamate attenuated the hepatic inflammatory response and apoptosis in suckling lambs with intrauterine growth retardation. Mediat Inflamm. 2020;2020:2453537. https://doi.org/10.1155/2020/2453537.

51. Sajnani K, Islam F, Smith RA, Gopalan V, Lam AK. Genetic alterations in Krebs cycle and its impact on cancer pathogenesis. Biochimie. 2017;135:164-72. doi:https://doi.org/10.1016/j.biochi.2017.02.008.

52. Al-Dhabi NA, Esmail GA, Duraipandiyan V, Valan Arasu M, Salem-Bekhit MM. Isolation, identification and screening of antimicrobial thermophilic Streptomyces sp. Al-Dhabi-1\&nbsp;isolated from Tharban hot spring, Saudi Arabia. Extremophiles Life Under Extreme Condi. 2016;20:79-90. https://doi.org/10.1007/s00792-015-0799-1.

53. Wen $\mathrm{C}$, et al. Metabolic changes in rat urine after acute paraquat poisoning and discriminated by support vector machine. Biomed Chromatogr. 2016;30:75-80. https://doi.org/10.1002/bmc.3627.

54. Li L, et al. Anti-inflammatory and gastrointestinal effects of a novel diclofenac derivative. Free Radic Biol Med. 2007:42:706-19. https://doi. org/10.1016/j.freeradbiomed.2006.12.011.

55. Baskar R, Sparatore A, Del Soldato P, Moore PK. Effect of S-diclofenac, a novel hydrogen sulfide releasing derivative inhibit rat vascular smooth muscle cell proliferation. Eur J Pharmacol. 2008;594:1-8. https://doi. org/10.1016/j.ejphar.2008.07.029.

\section{Publisher's note}

Springer Nature remains neutral with regard to jurisdictional claims in published maps and institutional affiliations. 